\title{
Expression Profile of Galectins (Gal-1, Gal-9, Gal-11 and Gal-13) in Human Bone Marrow Derived Mesenchymal Stem Cells in Different Culture Mediums
}

\author{
Faouzi Jenhani \\ Additional information is available at the end of the chapter \\ http://dx.doi.org/10.5772/55383
}

\section{Introduction}

Mesenchymal stem cells (MCSs) are refined as undifferencitaed cells that are capable of self renewal and differentiation into several cell types such chondrocyte, adipocyte osteocyte, myocyte and neuron-like cells. MSC can be isolated from bone marrow umbilical cord blood adipose tissue placenta. Although bone marrow(BM) has been regarded as a major source of MSC umbilical cord blood has been regarded as an alternative source for isolation of MSC. Human umbilical cord blood derived mesenchymal stem cell (hUCB-MSCs) have a capacity similar to that of BM-MCSs for multi-lineage differentiation

Researchers are interested in these cells because of their ability of differentiating into multiple mesenchymal and non mesenchymal lineages. Furthermore, MSCs evoke only minimal immunoreactivity and they display trophic, anti-inflammatory, and immunomodulatory capacities, through secretion of bioactive soluble factors with anti-inflammatory and immunomodulatory effects in vivo. These properties were confirmed by both experimental and clinical studies which demonstrated that the MSCs support hematopoiesis and enhance the engraftment of hematopoietic stem cells (HSC) after cotransplantation, which may contribute to a reduced incidence of graft versus host disease (GVHD). [1,2,3,4,5,6,7]

The galectins are a family of soluble lectins characterized by their affinity for b-galactoside residues. These proteins have recently attracted increasing attention because of theirinvolvement in various physiological and pathological processes. In addition, these proteins have recently attracted increasing attention of cancer biologists because of their essential functions including development, differentiation, cell-cell adhesion, cell-matrix interaction, growth 
regulation, apoptosis, RNA splicing, and tumor metastasis. Also, it has been shown that galectins' levels are altered in various cancers. [8,9,10]

Galectins-1,3,9 and 13 were among the best characterized members of this family. These galactins possesses several functions and were expressed in many tissues.

Given the importance of galectins, we investigated in this work, their expression by BM MSCs in different culture mediums

\section{Materiel and methods}

\subsection{Preparation of hBM-MSC}

MSCs were extracted and isolated from two sources adult the bone marrow. H- BM cells was harvested from the sternum or the iliac crest of healthy donors [20] after obtaining an informed consent was obtained in the Bone Marrow Graft National Center of Tunisia. The mean age of donors was $30 \pm 2$ years (range, 15-40 years).

MSCs were isolated using the classical plastic adhesion method. Mononuclear cells (MNCs) were isolated from hBM by density gradient (Ficoll Hypaque solution d=1. 077) centrifugation. After centrifugation at $800 \mathrm{~g}$ for $20 \mathrm{~min}$ at room temperature. MNC layer was removed from the interphase and washed twice with Hank's buffered salt solution and seeded into uncoated T25 or T75 flasks (Becton Dickinson, Bedford, MA, USA) at a cell concentration of $1 \times 10^{5}$ cells/ $\mathrm{cm}^{2}$ for BM cells and cultured in three condition mediums:

i. (M1) : basic growth medium consisting of alpha-Minimum Essential Mediums (alpha -MEM, Gibco BRL, Grand Island, NY, USA), supplemented with $10 \%$ (v/v) fetal bovine serum (Sigma-Aldrich, Bornem, Belgium),100U/mL penicillin, $0.1 \mathrm{mg} / \mathrm{mL}$ streptomycin (Gibco BRL), 2Mm L-glutamine (Gibco BRL), 0. $025 \mathrm{mg} / \mathrm{mL}$ fungizone (Gibco BRL) 10\% (v/v) of prescreened fetal bovine serum (FBS; Perbio Hyclone, Logan, Utah, USA) and $1 \mathrm{ng} / \mathrm{mL}$ fibroblast growth factor2 (FGF2) (AbCys, Paris, France)

ii. (M2): basic growth medium with fetal bovine serum and FGF2 replaced by $5 \%(\mathrm{v} / \mathrm{v})$ human patelet lysed (HPL)

iii. (M3): basic growth medium with fetal bovine serum and FGF2 replaced by $10 \%$ $(\mathrm{v} / \mathrm{v}) \mathrm{h}(\mathrm{HPL})$. Cell cultures were incubated at $37^{\circ} \mathrm{C}$ in a $5 \% \mathrm{CO} 2$ humidified atmosphere. The medium was changed twice weekly thereafter. When reaching $60 \%-80 \%$ confluence, the adherent cells were detached after treatment with $0.05 \%(\mathrm{v} / \mathrm{v})$ trypsin/ $1 \mathrm{mM}$ EDTA solution (Gibco BRL) and expanded by replating at a lower density at $10^{3}$ cells per $\mathrm{cm}^{2}$. All the studies were performed after 2 passages (P2).

\subsection{Induction of the differentiation directions}

We induced the differentiation of the MSCs when they reached the confluence. We used three differentiation mediums according to the focused differentiation line: 


\subsubsection{Osteogenic induction (O)for analysis galectins expression}

At $50 \%$ confluence, the cells were cultured for 14 days in DMEM-HG (High Glucose $4.5 \mathrm{~g} / \mathrm{L}$ ) containing 2\% FBS, $0.1 \mu \mathrm{M}$ dexamethasone (Sigma), $2 \mathrm{mM} \beta$-glycerolphosphate (Sigma), and $100 \mu \mathrm{M}$ ascorbate-2-phosphate (Sigma) with medium changes every 3 days. After 2 weeks of induction, the cells were stained according to Von Kossa's and Alizarin Red methods to detect the presence of calcium deposition into osteocytes.

\subsubsection{Adipogenic induction (A) for analysis galectins expression}

The adipogenic induction medium consisted of DMEM-LG (Low Glucose 1g/L) supplemented with $20 \%$ FBS, $1 \mu \mathrm{M} / \mathrm{L}$ dexamethasone, $0.5 \mathrm{mmol} / \mathrm{L}$ isobutylmethylxanthine (IBMX; Invitrogen), and $60 \mu \mathrm{mol} / \mathrm{L}$ indomethacin (Sigma). Adipogenic differentiation was evaluated after 2 weeks of induction, by the cellular accumulation of neutral lipid vacuoles that were stained with Nile Red (Sigma) and observed by fluorescent microscopy.

\subsubsection{Vascular smooth muscle induction (V) for analysis galectins expression}

Vascular smooth muscle (VSM) differentiation was obtained in a Mc Coy'5A medium supplemented with 12. 5\% FBS, 12. 5\% HS (Horse Serum, Invitrogen), $20 \mu \mathrm{M}$ L-glutamine, 0,8 $\mathrm{mM}$ L-serine, 0,15 mM L-asparagine, $1 \mathrm{Mm}$ sodium pyruvate, $5 \mathrm{mM}$ sodium bicarbonate, 1 $\mu \mathrm{M}$ hydrocortisone and amphotericin $\mathrm{B}$ and antibodies. The medium was changed every 4 days. The VSM differentiation was evaluated after 3 weeks of induction.

\subsection{Immunophenotyping of mesenchymal stem cells by flow cytometry}

MSCs were immunophenotypically characterized by flow cytometry using the following fluorochrome (FITC PE, PerCP) marked monoclonal antibodies anti : CD45, CD105, CD106, CD90,CD49, CD34, et CD73 and CD14 (Becton Dickinson and Company BD Biosciences San Jose CA).

\subsection{Detection of galectins (Gal-1, Gal-9 Gal-11 and Gal-13), by flow cytometry}

To reveal the expression of the galactin we used biotynaled antibodies against human recombinant galectin (Gal-1, Gal-9, Gal-11 and Gal-13). Thus we incubated 1,5 10 ${ }^{6}$ MSCs cells with the antibody which is specific to the focused galectin, for 30 minutes, at $4^{\circ} \mathrm{C}$. We incubated also the MSCs with a control antibody which doesn't recognize any protein, but has the same isotype to evaluate a background noise which correspond to non-specific fixation of the primary antibody.

The MSCs were washed 2 times with PBS 1X solution, then incubated for 30 minutes with Streptavidin coupled with a phycoerythrin (PE) fluorochrome phycoerythrin (PE). Flow cytometry analysis was performed on a FACS calibur, and data were analyzed using CellQuest software (Becton-Dickinson, BD Biosciences San Jose, CA). 


\subsection{Galectins immunofluorescence detection}

MSCs were fixed using $4 \%$ Methanol for 30 minutes at $+4^{\circ} \mathrm{C}$ and washed with PBS. The primary antibodies against many anti-antibodies: Gal-1-Streptavidine, Gal-3-Streptavidine, Gal-9Streptavidin and Gal-13 Streptavidin were diluted in 10\% BSA and 0.2\% Tween 20 (1:100) and were incubated at $4^{\circ} \mathrm{C}$ for $12 \mathrm{~h}$ followed by washings 3 times with PBS. For immunofluorescence non-specific binding sites were blocked with $10 \%$ BSA-PBS. For secondary immunofluorecence Biotine -fluoroscein were diluted in 10\% BSA-PBS and incubated for $60 \mathrm{~min}$ at $+4^{\circ} \mathrm{C}$ in the dark. Images were taken with camera and with inverse fluorescence microscopy

\subsection{RNA isolation and reverse transcription - Polymerase chain reaction before and after induction}

\subsubsection{RNA isolation}

For RT-PCR, Total RNA was extracted from cell culture in total confluence [90\% ) using TRIZOL reagent (Gibco BRL) according to the manufacturer's instructions. Reverse transcription was carried out using PrimeScript ${ }^{\text {TM}}$ RTase (Takara: Japan and Applied Biosystems) and the cDNA fragments were amplified using RNase Inhibitor (Takara: Japan and Applied Biosystems): For each reaction, we mixed $5 \mu \mathrm{l}$ of ARN or $5 \mu \mathrm{l}$ of distilled water (for the control), $6 \mu \mathrm{l}$ desoxyribonucleotides (dNTP)(TaKaRa Japan and Applied Biosystems), $1 \mu \mathrm{l}$ primers specific of the gene sequence (table 1) et $0.5 \mu$ l of enzyme la Taq polymerase enzyme (Takara: Japan and Applied Biosystems).

\subsubsection{RT-PCR before and after induction for the confirmation of the three directions of differentiation}

After denaturation at $65^{\circ} \mathrm{C}$ for $5 \mathrm{~min}$, amplification was carried out by 30 cycles at $30^{\circ} \mathrm{C}$ for 10 min, $42^{\circ} \mathrm{C}$ for $60 \mathrm{~min}$ and $95^{\circ} \mathrm{C}$ for $5 \mathrm{~min}$. Primers used are shown in Table 1 . The expression of the following genes were analyzed before (D0) and after differentiation (D14) into three directions of differentiation: osteogenesis $(\mathrm{O})$, adipogenesis $(\mathrm{A})$ and vascular smooth muscle (V). Glyceraldehyde 3-phosphate dehydrogenase (GAPDH) gene expression was used as control. The sequences of the oligonucleotides were reported in Table 1. Thermocycling was performed with a gradient thermocycler (Takara, Japan and Applied Biosystems). The analyse of the PCR products was carried out in an electrophoresis gel 1\% (Sigma).

\begin{tabular}{|c|c|c|}
\hline Construct & Sequence forward & Sequence reverse \\
\hline GAPDH & 5'- AATCCCATCACCATCTTCCAGG-3' & 5'-AGAGGCAGGGATGATGTTCTGG-3' \\
\hline PAL & 5'-CTGGACCTCGTTGACACCTG-3' & 5'-GACATTCTCTCGTTCACCGC-3' \\
\hline Runx2 & 5'-AACTTCCTGTGCTCGGTGCTG-3' & 5'-GGGGAGGATTTGTGAAGACGG-3' \\
\hline LPL & 5'-AAAGCCCTGCTCGTGCTGAC-3' & 5'-TAAACCGGGCCACATCCTGT-3' \\
\hline PPR & 5'-GGAGAAGCTGTTGGCGGAGA-3' & 5'-TCAAGGAGGCCAGCATTGTG-3' \\
\hline ASMA & 5'-TCATGATGCTGTTGTAGGTGGT-3' & 5'-CTGTTCCAGCCATCCTTCAT-3' \\
\hline
\end{tabular}

Table 1. Sequences of the primers used in RT-PCR study 
2.6.3. Reverse transcription - Polymerase chain reaction before and after induction for Galectin1,Gal-9, Gal-11 and Gal-13 expression

After denaturation at $65^{\circ} \mathrm{C}$ for $5 \mathrm{~min}$, amplification was carried out by 30 cycles at $30^{\circ} \mathrm{C}$ for 10 $\min , 42^{\circ} \mathrm{C}$ for $60 \mathrm{~min}$ and $95^{\circ} \mathrm{C}$ for $5 \mathrm{~min}$. Primers used are shown in Table 2 .

\begin{tabular}{ccc}
\hline Construct & Sequence forward & Sequence reverse \\
\hline GAPDH & 5'AATCCCATCACCATCTTCCAGG3' & 5'AGAGGCAGGGATGATGTTCTGG3' \\
\hline Gal 1 & 5'ATGGCTTGTGGTCTGGTC3' & 5'TCAGTCAAAGGCCACACA3' \\
\hline Gal 9 & 5'ATGGCCTTCAGCGGTTCC3' & 5'CTATGTCTGCACATGGGTCAG3' \\
\hline Gal 11 & 5'ATGAGTCAGCCCAGTGGG3' & 5'TCAGGAGTGGACACAGTAGAG3' \\
\hline Gal 13 & 5'ATGTCCCTGACCCACAG3' & 5'TCAATCGCTGATAAGCACT3' \\
\hline
\end{tabular}

Table 2. Sequences of the primers used in RT-PCR study

Thermocycling was performed with a gradient thermocycler (Takara, Japan and Applied Biosystems). The analyse of the PCR products was carried out in an electrophoresis gel $1 \%$ (Sigma).

\subsection{Statistical analysis}

Data are expressed as mean \pm standard error of the mean. Statistical comparisons were performed using the t-test student or Mann Whitney test with the program Graphpad, version. 5. A one-way analysis of variance (ANOVA) was done for paired samples; $p<0.05$ was considered to be statistically significant.

\section{Results}

\subsection{Morphologic analysis of MSCs derived from bone marrow in terms of culture mediums}

To prepare MSCs cultures, we isolated mononuclear cells (MNCs) from bone marrow (hBM). The MNCs derived from bone marrow were put in a density of $1.10^{6}$ cells $/ \mathrm{cm}^{2}$.

Adherent cell populations from the MNC fraction of $\mathrm{hBM}$ samples were generated by expansion culture using 3 different media: (M1) Alpha MEM with 10\% FBS and $1 \mathrm{ng} / \mathrm{mL}$ $\mathrm{FGF}_{2}$ (M2) Alpha MEM with 5\% HPL, (M3) Alpha with 10\% HPL. After two weeks of culture, an adherent and stable cell layer was obtained from BM derived MNC with all medium Mean time for the primary culture to reach subconfluence was 15 days. After one passage(P1), adherent cells displayed a fibroblast-like morphology in culture plate $(n=20)$. The figure 1 showed a particular morphology of cultured MSC. 


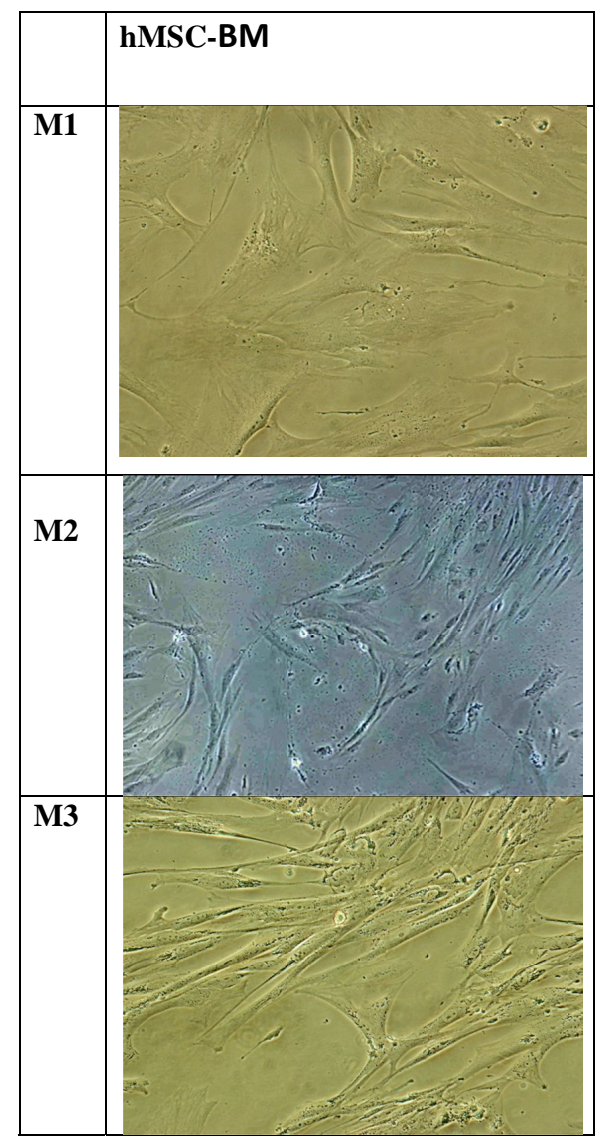

Figure 1. Morphological characterization of hMSC-BM cultured in different media in the P2 (passage): M1: (10\% FBS +FGF2), M2:(5\% HPL), M3:(10\% HPL). 


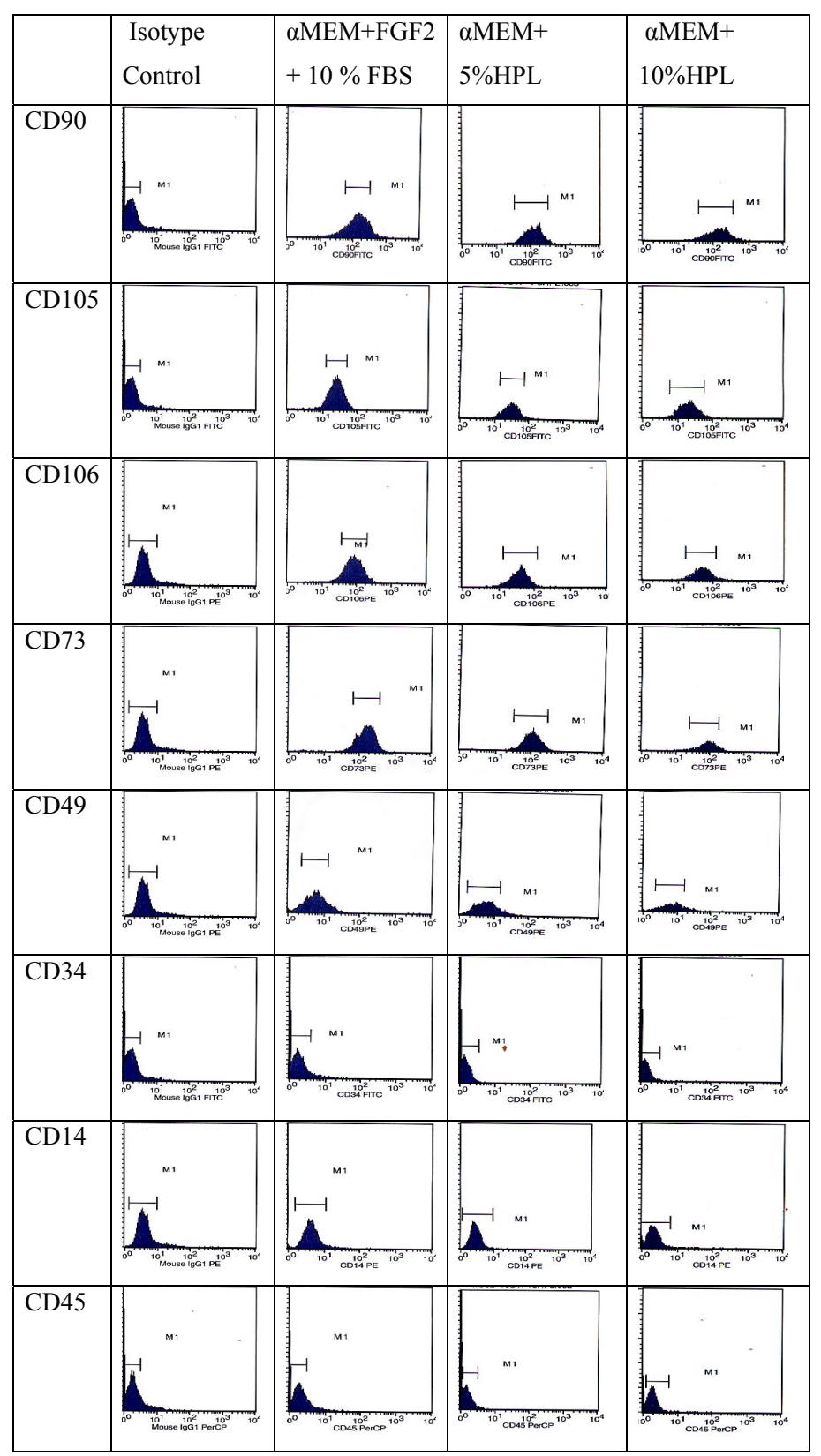

Figure 2. Representative flow cytometry analysis of BM-MSCs.Comparison of membrane antigen expression of BMMSCs cultured at P2 in different media: M1 :(10\% FBS+FGF $)$ M2: (5\% HPL), M3: (10\% HPL) 
Also MSCs were immunophenotypically characterized by flow cytometry. This analysis revealed that MSCs were uniformly positive for CD73 CD90 CD105 CD106 but negative for CD14 CD34 and CD45 (figure 2).

The influence of HPL or FBS on the osteogenic and adipogenic differentiation potential of MSCs was investigated after appropriate induction at the second passage (P2). Cells grown in HPL or FBS deposited an extensive mineralized matrix when cultured for 2 weeks in an osteogenic medium [ $2 \%$ FBS), as demonstrated by strong alizarin red staining. These cells also efficiently differentiated into the adipogenic lineage, as indicated by Oil Red O staining of lipid droplets in the cytoplasm following culture in an adipogenic medium.

All populations of BM-MSCs cultured in different conditions with the HPL showed osteogenic, Vascular smooth muscle and chondrogenic differentiation capacity. The difference appears in adipogenic differentiation with 10\% HPL (Fig 3 ).

Osteogenic, adipogenic, and vascular smooth muscular

differentiation capacity assessed by staining of BM-derived MSCs

Morphological

hBM MSCs
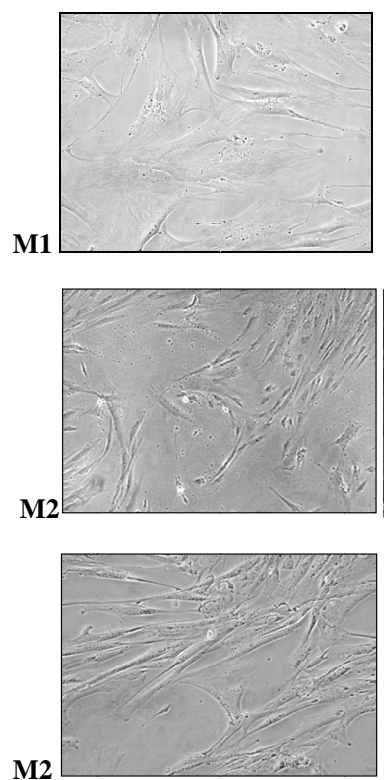

\section{Alizarin Red}

(0)
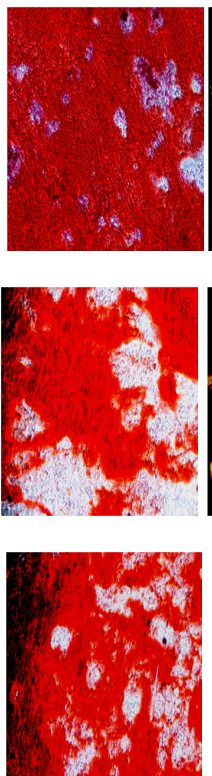

Nil Red

(A)
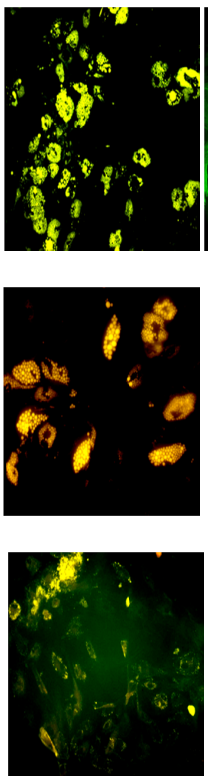

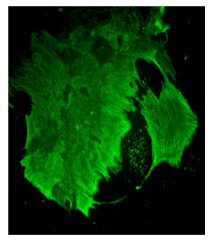

ASMA

(VSM)
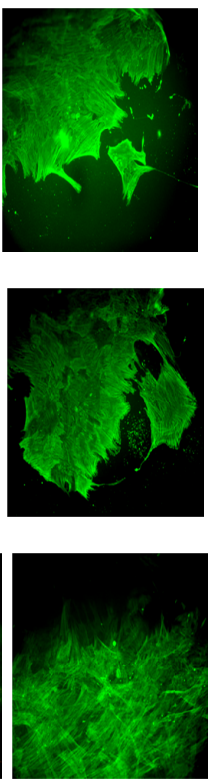

Figure 3. Morphological characterization of hBM- MSCs derived cultured in different media M1, M2 and M3 at P2 and Osteogenic, chondrogenic, adipogenic, and vascular smooth muscular differentiation capacity assessed by staining 
To confirm their differentiation potential, after P1 MSCs were plated in specific induction mediums to generate for adipocytes, osteoblasts, or chondrocytes.

To select the differentiated MSCs, we used the RT-PCR to search markers specific for the three differentiation directions. These markers are the alkalin phosphatase(PAL) as osteoblast specific marker the lipoprotein lipase (LPL)as specific of the adipocyte line and the actin-ASMA specific of vascular smooth muscles line. The positive control is the GAPDH. These markers were not expressed before the induction of the differentiation and presented an overexpression after the $14^{\text {th }}$ day from the induction of specific differentiation in the three expansion mediums (M1, M2, et M3) demonstrating the multipotent nature of the MSCs (figure 4).

A

BM (D0)
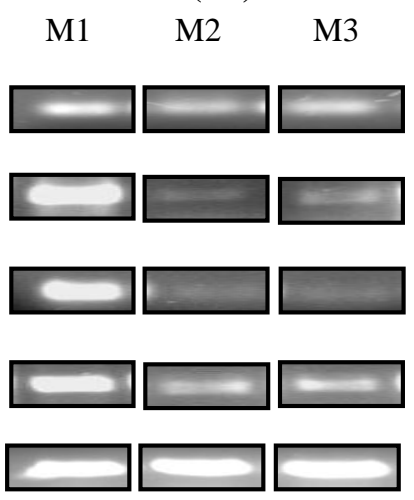

\section{ASMA}

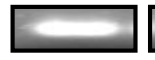

PPR $\gamma$
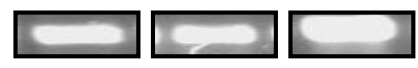

Runx 2
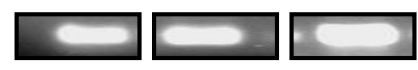

PAL
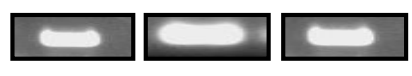

GAPDH
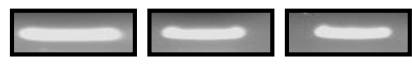

Figure 4. RT-PCR analyses of vascular smooth muscle (ASMA), adipogenic (PPR ), and osteogenic (Runx2, PAL) markers prior to (D0) (A) and after 14 days of differentiation induction (D14) (B) of BM-derived MSCs previously cultured in expansion media (M1, M2, M3)

\subsection{Detection of galectins in MSCs in terms of the culture mediums by flow cytometry}

MSCs from different BM samples were characterized by flow cytometry with a panel of biotynaled antibodies against human recombinant galectin (Gal-1, Gal-9, Gal-11 and Gal-13) at P2 after culture in the three mediums (M1, M2, et M3). Flow cytometry analysis revealed that MSC derived-hBM constitutively expressed galectins (Gal-1, Gal-9, Gal-11, Gal-13) at 
different mediums but with unequal percentages. We noticed that galectins 9 and 11 are more expressed by cells derived from hBM with an average of [88.1 $\pm 1.8 \%$ ) in M1 Meduim in comparison to M2 $(16,8 \pm 3.9 \%)$ and M3 $(11,6 \pm 3.9 \%)$ with $\mathrm{p}<0.01$.

We noticed also that galectin 1 is expressed with in average of $(91.2 \pm 1.4 \%)$ at P2 after culture in the three mediums (M1,M2,M3). In the contrary no expression of galactin 13 have been noted in any medium. (figure 5)

\begin{tabular}{|c|c|c|c|c|c|c|}
\hline \multirow[b]{3}{*}{$\begin{array}{l}\text { Anti- } \\
\text { Gal } 1\end{array}$} & \multicolumn{2}{|l|}{$\begin{array}{l}\text { Standard medium } \\
\text { ( MEM + FGF) : M1 }\end{array}$} & \multicolumn{2}{|c|}{ Medium with $5 \% \mathrm{HPL}$ : M2 } & \multicolumn{2}{|c|}{ Medium with $10 \% \mathrm{HPL}: \mathrm{M} 3$} \\
\hline & Isotyope control & Anti-Gal & Isotyope control & Anti-Gal & Isotyope control & Anti-Gal \\
\hline & 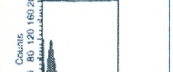 & & 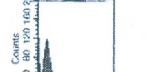 & & 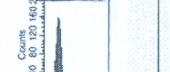 & \\
\hline $\begin{array}{l}\text { Anti- } \\
\text { Gal } 9\end{array}$ & 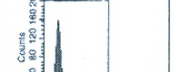 & & 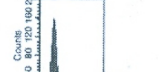 & & & \\
\hline $\begin{array}{l}\text { Anti- } \\
\text { Gal } 11\end{array}$ & 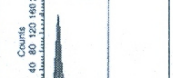 & & 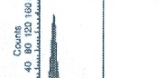 & 资 & & \\
\hline $\begin{array}{l}\text { Anti- } \\
\text { Gal } 13\end{array}$ & 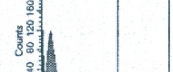 & 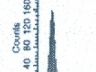 & 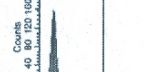 & 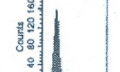 & 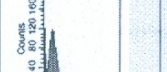 & 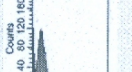 \\
\hline
\end{tabular}

Figure 5. Galectins Gal-1, Gal-9,Gal-11 and Gal-13 antigen expression of BM-MSCs cultured at P2 in different media: M1 (10\% FBS+FGF2) M2(5\% HPL), M3 (10\% HPL) by flow cytometry

\subsubsection{Expression of galectins of MSC-BM by microscopy immunofluorecence}

Immunofluorecsence of galectin-1 in MSC of normal adult bone marrow was showed expression in three mediums M1,M2,M3. Following this technique, we are also identified galectins gal-9 and gal-11 expression but only in standard medium M1. No expression of gal- 13 have been reveled by hBM-CSM cultured in all media M1,M2 and M3. we are determined the localization of galectin mostly in nucleus cells. (Fig6). 


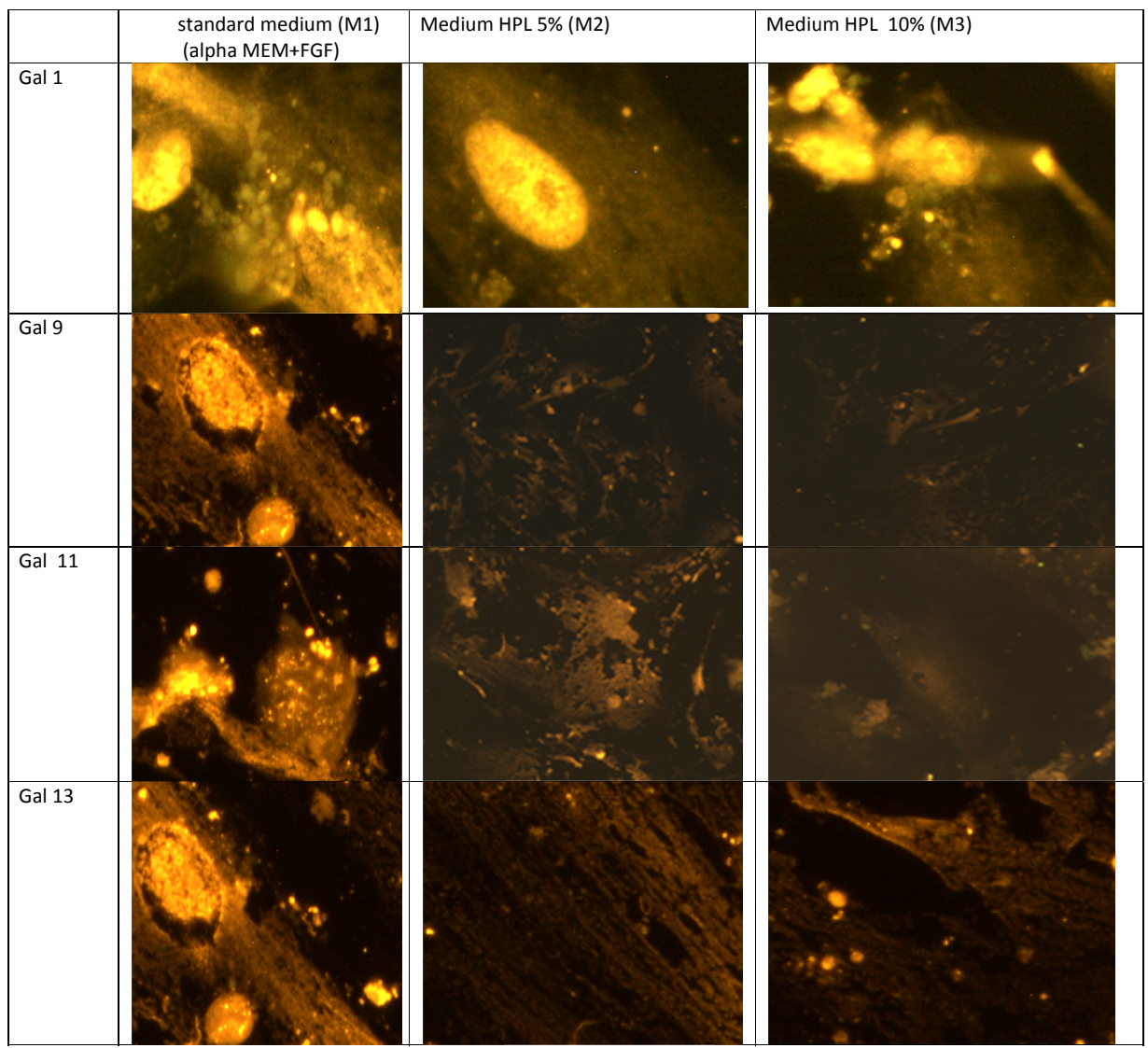

Figure 6. Immunofluorescence Galectin-1, Galectin-9, Galectin -11 and Galactin-13 expression by cultured at P2 in different media: M1 (10\% FBS+FGF $)$ M2 (5\% HPL), M3 (10\% HPL) 


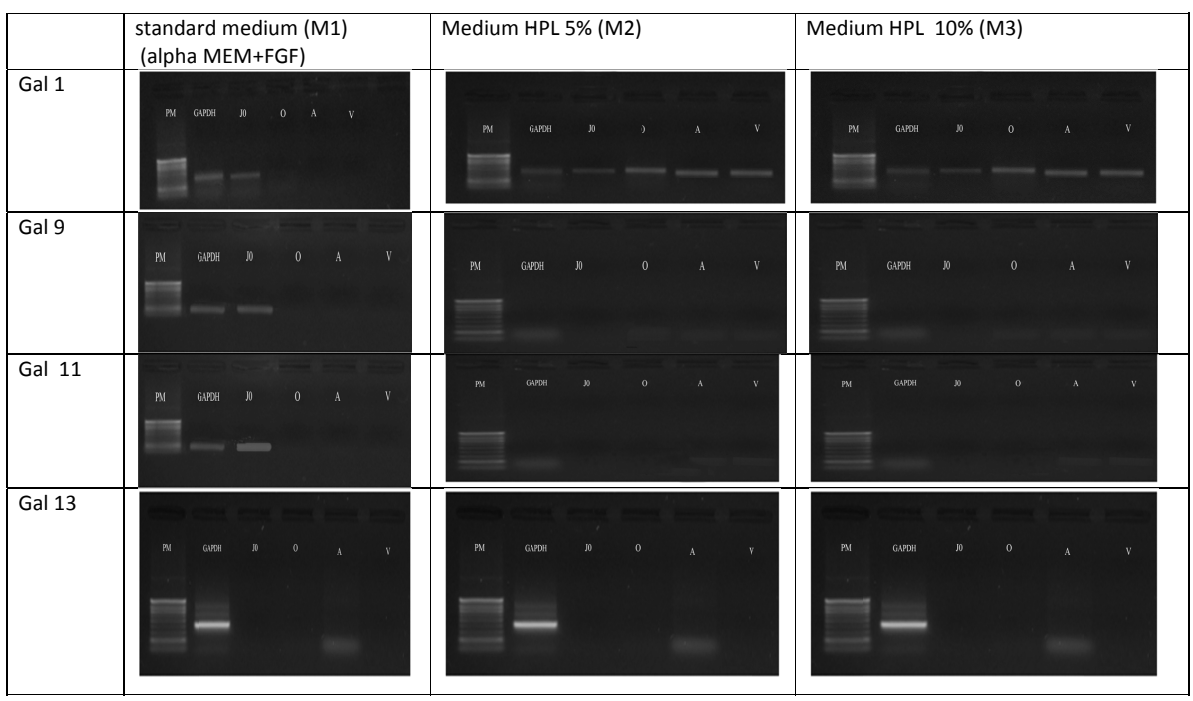

Figure 7. RT -PCR Galectin-1, Galectin-9, Galectin -11 and Galactin-13 expression by cultured at P2 in different media: M1 (10\% FBS+FGF $)$ M2 (5\% HPL), M3 (10\% HPL) and after differentiation process ( O: osteogenic induction, A: Adipogenic induction,V: Vascular Smooth Muscle induction, GAPDH : Glyceraldheyde 3-phospate dehydrogenase, PM: Weight molecular )

\subsection{RT -PCR galectin-1,Gal-9,Gal-11 and Gal-13 expression by hBM-MSCs}

By RT-PCR, we evaluated the messenger RNA expression of Galectin-1,Galectin-9, Galectin-11 and Galectin -13 in hBM-MSCs before and after differentiation to the three direction : osteoblastic [0), adipocyte (A) and to vascular smooth muscle (VSM).

We had a positive results for hBM-MSCs both before their differentiation and after the induction of the three differentiation directions:

- before the induction of the differentiation process :Gal-1 was expressed by hBM- MSCs in the standard medium M1 (MEM+ FGF2) and also in the three mediums (M2, M3, et M4) which contain the human platelet lysat (HPL). While, after the induction of the MSCs differentiation, Gal-1 was only expressed in MSCs cultivated in mediums containing the HPL (M2, M3), in the three differentiation directions (O, A, VML)

- Concerning the expression of Gal-9 by BM-MSCs: we observed that Gal-9 was expressed by undifferentiated BM-MSCs cultivated in the standard medium (M1: MEM+ FGF2), but absent in MSCs cultivated in mediums containing the HPL (M2, M3). However, after inducing the differentiation process, this galectin was absent in MSCs of the standard medium and had a very low expression in mediums containing the HPL (M2, M3).

- Regarding the expression of Gal-11 by BM-MSCs, we found that Gal- 11 was expressed by undifferenciated BM-MSCs, in the standard medium M1 (MEM+ FGF2) but absent in BM- 
MSCs cultivated in M2, M3, and M4. And After the MSCs differentiation Gal-11 was no more expressed in the standard culture's medium (M1 : MEM+ FGF2), while MSCs cultivated in mediums containing the HPL (M2, M3) expressed the Gal-11 in adipocytes (A) and vascular smooth muscle cells (VML). As to Gal-13, we reported that this galectin wasn't expressed by the undiferenciated MSCs neither in the standard medium nor in the mediums containing the HPL (M1, M2, and M3). Whereas, after the MSCs differentiation, Gal-13 was only expressed by adipocytes. (figure 5)

\section{Discussion}

Multipotent mesenchymal stromal cells (MSCs) have generated a great debate as a potential source of cells for cell-based therapeutic strategies primarily owing to their intrinsic ability to self renew and differentiate into functional cell types that constitute the tissue in which they exist and also because MSCs express a large number of molecules including the galectins. [11,12]

The galectins are a family of soluble lectins characterized by their affinity for $\beta$-galactoside residues. In recent years, galectins have become a major focus of investigation because of their involvement in various physiological and pathological processes. [13,14]

Given that galectins have been shown to have important effects $[13,14]$ we decided to evaluate the expression of galectins (Gal-1, Gal-3, Gal-9 and Gal-13) by MSCs.

In this study we analyzed MSCs cultured and expanded in three conditions mediums: basic growth medium consisting of alpha-Minimum Essential Medium in which HPL replaced FBS in order to investigate their morphology, plasticity, proliferation differentiation to osteoblasts, adipocytes, and vascular smooth muscles and their capacity to express galectins in terms of the mediums' compositions. [15,16]

In our laboratory MSCs were characterized and defined according to the International Society for Cellular Therapy minimal criteria. [17,18]. So first, we demonstrated that BM-MSC adhered to plastic. Second as measured by flow cytometry all of MSC derived from BM-MSC were nearly $100 \%$ positive for the makers CD90 CD105, and CD73 ( $\geq 95 \%)$, while they lack the expression of CD45 CD34, and CD14 $(\leq 2 \%)$. Third, BM-MSC differentiated to osteoblasts adipocytes and vascular smooth cells.

First, we investigated the effect of culture mediums on the adherence and the proliferation of MSCs. Then we demonstrated that the three mediums allow the adherence of MSCs to plastic and their proliferation, but the percentage of confluence and the times it takes to get it, change in terms of the composition of the culture medium. So, the culture mediums containing $5 \% \mathrm{HPL}$ induced the best MSCs confluence. Thus the most important confluence of the bone marrow's MSCs was reached at the first passage (P1) in the culture's medium 5\% HPL after 30 days from their implantation. So, we confirmed that HPL-expanded MSCs show a significantly higher proliferation rate, in comparison with MSCs expanded in the standard medium. So, we can use the HPL as a substitute of FBS in mediums specific to the culture of MSCs destined for clinical applications, to avoid a possible xenogenic contamination caused by the FBS. [19] 
The expansion-promoting effect of the HPL is likely to result from the high concentration of natural growth factors that HPL contains. In fact platelet granules contain many growth factors, including platelet-derived growth factor (PDGF), fibroblast growth factor (FGF), insulin-like growth factor (IGF), transforming growth factor (TGFb), platelet factor 4 (PF-4), and plateletderived epidermal growth factor(PDEGF) [20,21]. Thesegrowth factors, which are released from platelet lysate, have been shown to enhance MSC expansion in vitro. [20, 21,22,23,24]

Second, we analyzed the morphology of MSC, and observed fibroblast-like cells in BM. Thus the MSCs formed a monolayer of homogenous spindle-shaped cells with a whirlpool-like array. This result is in accordance with the literature. [25]. However, two cell phenotypes were observed among fibroblast-like BM-MSC colonies: thin spindle shaped MSC and star-shaped MSC. These BM-MSC two phenotypes were described by Wolgang et Wagnera and al [25]

To detect the expression of galectins Gal-1, Gal-9, Gal-11 and Gal-13 by MSCs, we used two techniques which are the flow cytometry (FC) and the RT-PCR. We searched the expression of galectins in MSCs cultivated in the three culture mediums: the standard medium: alphaMEM+ FGF2, the medium (with 5\% HPL), the medium (with 10\% HPL).

The flow cytometry revealed that all the galectins of interest (Gal-1, Gal-3, Gal-9, and Gal-13) are expressed in BM-MSCs, in above mentioned culture mediums,. It thus becomes clear that expression percents of galacetins revealed by the flow cytometry in hBM-MCSs depend on two parameters which are the composition of the medium and the type of the galectin.

Thus, we observed mainly that the best Galectins' expression was visualized in the standard medium (alpha-MEM /FGF2). Previous studies have shown that the supplementation of fibroblast growth factor 2 (FGF2) in vitro selects for the survival of a large number of of MSCs enriched in pluripotent mesenchymal precursors enhances the growth of MSCs maintains their multilineage differentiation potential during in vitro expansion and prolongs their life span [26]. So, this data and our observation suggest that the increase of galectins in the presence of FGF2 is due to the improvement of the growth the proliferation and differentiation potential of MSCs.

Then, when comparing the means of the galectins' expression by the MSCs we concluded that the best means of protein expression were those of Gal-1; this galectin had the highest means of expression in all culture mediums. Friederike Gieseke and al. confirm our result; thus, they report that Gal-1 is highly expressed in MSCs. [27]

The results of the RT-PCR are in accordance with those of the flow cytometry. Thus, the RTPCR applied to RNA of the MSCs revealed that galectins (Gal-1, Gal-9, Gal-11, and Gal-13) are expressed by both undifferentiated and differentiated BM-MSCs. So, before the induction of the differentiation process, the MSCs cultivated in the standard medium expressed Gal-1, Gal-9, Gal-11, but not Gal-13, while the MSCs cultivated in the mediums enriched by the HPL expressed only the Gal-1. Nevertheless, after the induction of the MSCs differentiation, first we observed that Gal-1, Gal-9, Gal-11 were no more expressed in the standard culture's medium (alpha MEM+ FGF2) while we detected the expression of Gal-13 in the adipocytes. Second, when examining the expression of galectins by MSCs cultivated in mediums containing the HPL we noticed the presence of Gal-1, Gal-9 with a low expression, Gal-11 in both adipocytes and VMLs and finally Gal-13 in adipocytes. 
We noted that MSCs expressed the galectins constitutively, but this expression was modulated differently depending on the type of the galectin the culture's stage and the composition of the culture medium.

These findings are in agreement with previous reports. Thus, Friederike Gieseke and al. [27] claim that $m$ RNAs of Gal-1, Gal-3, -8 and -9 were detected in MSCs by the RT-PCR and when performing a quantitative real time -PCR the m RNAs of Gal-3, -8 and -9 were detected to a lesser extent than Gal-1 which is similar to the results we obtained when performing the flow cytometry.

Najar M and al. [14] confirmed the constitutive expression of galectin-1 m RNA by BM-MSCs. Regarding Gal-3 Ju-Yeon Kim and al. [28] demonstrated that Gal-3 is secreted by UCB-MSC under pathological conditions whereas low level of GAL-3 was detected by Western blot analysis in conditioned medium of naïve UCB-MSC. This report is in contrast with our results since we confirmed the absence of galectins' in both membranes and RNAs of UC-MSCs.

The present study provides new insights concerning the expression of the galectins by the MSCs. According to our observations and the reports mentioned above the galectins are located both in nuclear and membrane levels. This suggests that the galectins are released by MSCs. Because of their their multilineage differentiation potential, immunomodulatory and anti-inflammatory properties, MSCs have become increasingly attractive as a therapeutic approach. Careful characterization of MSC physiology and the effects of culture mediums context on their proliferation differentiation and molecules' secretion will increase the safety and efficiency of MSCs in clinical settings.

\section{Author details}

Faouzi Jenhani ${ }^{1,2^{*}}$

Address all correspondence to: faouzi.jenhani@yahoo.tn, tunisiacelltherapy@ymail.com

1 Cell Immunology and Cytometry and Cell Therapy Laboratories, Blood National Center, Tunisia

2 Unit Immunology Research, Faculty of pharmacy, Monastir, Tunisia

\section{References}

[1] Tse, W. T. , Pendleton, J. D. , Beyer, W. M. , Egalka, M. C. , Guinan, E. C. Suppression of allogeneic T-cell proliferation by human marrow stromal cells: implications in transplantation. Transplantation,2003, 75, 389-397. 
[2] Selmani Z, Naji A, Zidi I, Favier B, Gaiffe E, Obert L, et al. Human leukocyte antigenG5 secretion by human mesenchymal stem cells is required to suppress T lymphocyte and natural killer function and to induce CD4 1 CD25 high FOXP31 regulatory T cells. Stem Cells 2008;26:212-222.

[3] Le Blanc K, Rasmusson I, Sundberg B, Gotherstrom C, Hassan M, Uzunel M, et al. Treatment of severe acute graft-versus-host disease with third party haploidentical mesenchymal stem cells. Lancet. 2004;363:1439-1441.

[4] Frank MH, Sayegh MH. Immunomodulatory functions of mesenchymal stem cells. Lancet. 2004; 363:1411-2.

[5] Danguy, A. , Camby, I. , Kiss, R. , 2002. Galectins and cancer. Biochim. Biophys. Acta $1572,285-293$.

[6] Yagi H, Soto-Gutierrez A, Parekkadan B, Kitagawa Y, Tompkins RG, Kobayashi N, Yarmush ML: Mesenchymal stem cells: mechanisms of immunomodulation and homing. Cell Transplant 2010, 19:667-679.

[7] Pittenger MF, Mackay AM, Beck SC, Jaiswal RK: Multilineage potential of adult human mesenchymal stem cells. Science 1999, 284:143-147.

[8] Maria Virginia Tribulatti, Galectin-8 provides costimulatory and proliferative signals to T lymphocytes. Journal of Leukocyte Biology 2009,Volume 86, August.

[9] Rabinovich GA. Galectins: an evolutionarily conserved family of animal lectins with multifunctional properties; a trip from the gene to clinical therapy. Cell Death Differ Aug 1999; 6(8):711-721.

[10] Camby I, Le Mercier M, Lefranc F, Kiss R. Galectin-1: a small protein with major functions. Glycobiology. 2006;16:137R-157R.

[11] D. Baksh, L. Song R. S. Tuan. Adult mesenchymal stem cells : Characterization differentiation and application in cell and gene therapy. J. Cell. Mol. Med. Vol 8, No 3, 2004 pp. 301-316.

[12] Deans RJ, Moseley AB. Mesenchymal stem cells: biology and potential clinical uses. Exp Hematol. 2000;28:875-884.

[13] Camby I, Le Mercier M, Lefranc F, Kiss R. Galectin-1: a small protein with major functions. Glycobiology. 2006;16: 137R-157R.

[14] Najar et al : Mehdi Najar, Gordana Raicevic, Hicham Id Boufker, Basile Stamatopoulos, Ce'cile De Bruyn, Nathalie Meuleman, Dominique Bron, Michel Toungouz, and Laurence Lagneaux. Modulated expression of adhesion molecules and galectin-1: Role during mesenchymal stromal cell immunoregulatory functions. Experimental Hematology 2010;38:922-932.

[15] Capelli C, Domenghini M, Borleri G, Bellavita P, Poma R, Carobbio A, et al. Human platelet lysate allows expansion and clinical grade production of mesenchymal stro- 
mal cells from small samples of bone marrow aspirates or marrow filter washouts. Bone Marrow Transplant 2007;40(8):785-91.

[16] Doucet C, Ernou I, Zhang Y, Llense JR, Begot L, Holy X, et al. Platelet lysates promote mesenchymal stem cell expansion: a safety substitute for animal serum in cellbased therapy applications. J Cell Physiol 2005;205(2):228-36.

[17] M Dominici K Le Blanc I Mueller I Slaper-Cortenbach FC Marini DJ Prockop and EM Horwitz. Minimal Criteria for defining multipotent mesenchymal stromal cells. The international society for cellular therapy position statement. Cytotherapy (2006) Vol 8, No. 4, 315-317.

[18] Horwitz EM, Le Blanc K, Dominici M, et al. Clarification of the nomenclature for MSC: The International Society for Cellular Therapy position statement. Cytotherapy. 2005; 7:393-395.

[19] Berger,M. G. ,Veyrat-Masson,R. ,Rapatel,C. ,Descamps, S. , Chassagne,J. ,BoiretDupre,N. ,2006. Cellculture medium compositionandtranslationaladultbone marrowderived stemcellresearch. StemCells24 (12), 2888-2890.

[20] Harrison P, Cramer EM. Platelet alpha-granules. Blood Rev 1993;7(1):52-62.

[21] Kilian O, Flesch I, Wenisch S, Taborski B, Jork A, Schnettler R, et al. Effects of platelet growth factors on human mesenchymal stem cells and human endothelial cells in vitro. Eur J Med Res 2004;9(7):337-44.

[22] Van den Dolder J, Mooren R, Vloon AP, Stoelinga PJ, Jansen JA. Platelet-rich plasma: quantification of growth factor levels and the effect on growth and differentiation of rat bone marrow cells. Tissue Eng 2006;12(11):3067-73.

[23] Capelli C, Domenghini M, Borleri G, Bellavita P, Poma R, Carobbio A, et al. Human platelet lysate allows expansion and clinical grade production of mesenchymal stromal cells from small samples of bone marrow aspirates or marrow filter washouts. Bone Marrow Transplant 2007;40(8):785-91.

[24] Doucet C, Ernou I, Zhang Y, Llense JR, Begot L, Holy X, et al. Platelet lysates promote mesenchymal stem cell expansion: a safety substitute for animal serum in cellbased therapy applications. J Cell Physiol 2005;205(2):228-36.

[25] Comparative characteristics of mesenchymal stem cells from human bone marrow, adipose tissue, and umbilical cord blood. Wolfgang Wagnera, Frederik Weina, Anja Seckingera, Maria Frankhauserb, Ute Wirknerc, Ulf Krausea, Jonathon Blakec, Christian Schwagerc, Volker Ecksteina, Wilhelm Ansorgec, and Anthony D. Hoa. Experimental Hematology 33 (2005) 1402-1416

[26] Bianchi et al : Ex vivo enrichment of mesenchymal cell progenitors by fibroblast growth factor 2. Giordano Bianchi,a,b,c,2 Andrea Banfi,a,b,c,1,2 Maddalena Mastrogiacomo,a,b,c Rosario Notaro,a Lucio Luzzatto,a Ranieri Cancedda,a,b,c and Rodolfo Quartoa,b,c,*. Experimental Cell Research 287 (2003) 98-105. 
[27] Friederike Gieseke and al : Friederike Gieseke, Judith Böhringer Rita Bussolari Massimo Dominci Rupert Handgretinger and Ingo Müller. Human multipotent mesenchymal stromal cells employ galectin-1 to inhibit immune effector cells. Jul 19, 2010; doi: 10. 1182/Blood-2010-02-270777.

[28] Ju-Yeon Kim and al: Galectin-3 secreted by human umbilical cord blood-derived mesenchymal stem cells reduces amyloid-b42 neurotoxicity in vitro Ju-Yeon Kim a,b, Dong Hyun Kim a, Dal-Soo Kim a, Ji Hyun Kim a, Sang Young Jeong a, Hong Bae Jeon a, Eun Hui Lee b, Yoon Sun Yang a, Wonil Oh a, Jong Wook Chang a, ${ }^{*}$ FEBS Letters 584 (2010) 3601-3608. 